\title{
Epizootiology of the parasitic dinoflagellate Hematodinium sp. in the American blue crab Callinectes sapidus*
}

\author{
Gretchen A. Messick ${ }^{1, * *}$, Jeffrey D. Shields ${ }^{2}$ \\ ${ }^{1}$ National Ocean Service, Center for Coastal Environmental Health and Biomolecular Research, \\ Cooperative Oxford Laboratory, 904 S. Morris St., Oxford, Maryland 21654-9724, USA \\ ${ }^{2}$ Virginia Institute of Marine Science, The College of William \& Mary, PO Box 1346, Gloucester Point, Virginia 23062, USA
}

\begin{abstract}
Hematodinium sp. is a parasitic dinoflagellate that infects and kills blue crabs Callinectes sapidus. Periodic outbreaks of dinoflagellate infections with subsequent high host mortalities prompted a study of the epizootiology and distribution of the crab pathogen. Hemolymph samples from over 13000 crabs were assessed for infections over 8 yr. Moderate to high prevalences were found at several locations along the Atlantic and Gulf coasts of the United States. In the coastal bays of Maryland and Virginia, prevalence followed a seasonal pattern, with a sharp peak in late autumn. Infections were significantly more prevalent in crabs measuring less than $30 \mathrm{~mm}$ carapace width; host sex did not influence prevalence. Prevalences were highest in crabs collected from salinities of 26 to $30 \%$; no infected crabs were found in salinities below $11 \%$. Intensity of infection did not vary among crab sizes, molt stages, or sexes. Naturally and experimentally infected crabs died over 35 and 55 d in captivity, with a mean time to death of approximately 13 and $42 \mathrm{~d}$, respectively. Several other crustaceans, including gammaridean amphipods, xanthid (mud) crabs, and the green crab Carcinus maenus, were found with Hematodinium-like infections. Considering its widespread distribution and high pathogenicity, we suggest that Hematodinium sp. represents a significant threat to blue crab populations in high salinity estuaries along the Atlantic and Gulf coasts of the USA.
\end{abstract}

KEY WORDS: Hematodinium sp. · Callinectes sapidus · Seasonality · Size $\cdot$ Salinity $\cdot$ Disease · Dinoflagellate Resale or republication not permitted without written consent of the publisher

\section{INTRODUCTION}

The blue crab Callinectes sapidus Rathbun supports valuable commercial fisheries along the Atlantic and Gulf coasts of the United States. Annual landings of blue crabs exceeded 97 metric tons from 1989 to 1993 , with US dockside values in 1994 estimated at \$137 million (Johnson et al. 1998). Blue crabs sustain the largest

\footnotetext{
*Disclaimer: The mention of trade names does not reflect endorsement by the National Ocean Service nor Virginia Institute of Marine Science

${ }^{* *}$ E-mail: gretchen.messick@noaa.gov
}

extant fishery in Chesapeake Bay. Outbreaks of disease caused by the parasitic dinoflagellate Hematodinium sp. in blue crabs have been reported in several coastal states (Newman \& Johnson 1975, Couch \& Martin 1982, Messick 1994). In the laboratory, experimentally infected blue crabs suffer high mortality rates $(>86 \%$ ) to the resultant disease, a level 7 to 8 times higher than uninfected controls (Shields \& Squyars 2000). Current models project crab abundance based on constant low levels of natural mortality (Lipcius \& Van Engel 1990, Abbe \& Stagg 1996, Rugolo et al. 1998). They do not consider the potential epizootics and resulting mortalities caused by Hematodinium sp. or other diseases. 
Members of the genus Hematodinium are parasitic syndinid dinoflagellates that invade the hemolymph and other tissues of their crustacean hosts (Shields 1994). Several commercially important crustaceans have been reported infected with Hematodinium spp. Epizootics have damaged fisheries for the Tanner crab Chionoecetes bairdi in Alaska (Meyers et al. 1987, 1990), the snow crab C. opilio in Alaska and Newfoundland (Meyers et al. 1990, 1996, Taylor \& Khan 1995), the Norway lobster Nephrops norvegicus in Scotland (Field et al. 1992), the velvet swimming crab Necora puber in France (Wilhelm \& Mialhe 1996), and the blue crab Callinectes sapidus in the USA (Newman \& Johnson 1975, Messick 1994). Other commercial species are also hosts to Hematodinium sp. infections, including rock crabs Cancer irroratus and $C$. borealis (MacLean \& Ruddell 1978), the Australian blue crab Portunus pelagicus, and the mangrove crab Scylla serrata (Shields 1992, Hudson \& Shields 1994). Infections also occur in lady crabs Ovalipes ocellatus (MacLean \& Ruddell 1978), and obligate coral-dwelling crabs Trapezia spp. (Hudson et al. 1993). Hematodinium-like dinoflagellates also occur in amphipods (Johnson 1986), and dinoflagellate-like infections occur in spot shrimp Pandalus platyceros (Meyers et al. 1994).

Hematodinium perezi was first reported as a rare parasite in green crabs Carcinus maenus from Europe (Chatton \& Poisson 1931). Newman \& Johnson (1975) identified the dinoflagellate in blue crabs as $H$. perezi, based on similarities in morphological characteristics reported by Chatton \& Poisson (1931). The dinoflagellate in blue crabs we previously identified as $H$. perezi (Messick 1994, Shields \& Squyars 2000) is not designated a species in this report. Additional molecular and morphological comparisons must be made between the type species, $H$. perezi in the green crab from the type locality (France) and the blue crab parasite, before assigning a species identification.

The goal of this study was to clarify the potential threat of Hematodinium sp. infections to blue crab populations. We report the distribution and prevalence of the parasite along the Atlantic and Gulf coasts of the United States, document seasonality of infections, potential relationships between infections and several host factors, relate the distribution and epizootics of the parasite to hydrographic features where infections progress and spread, and identify several potential reservoir hosts for the parasite.

\section{MATERIALS AND METHODS}

Sampling concentrated on crabs from waters surrounding the Delmarva Peninsula to obtain seasonal and long-term data. In Maryland (MD), blue crabs were collected semimonthly, from 1991 through 1997, from 21 stations within the coastal bays, in conjunction with the MD Department of Natural Resources Coastal Bay Fisheries Project. Crabs were collected with a

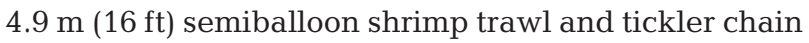
towed for 6 min at 3 knots. The trawl had a $3.8 \mathrm{~cm}$ (1.5 inch) mesh in the body, a $3.2 \mathrm{~cm}$ (1.25 inch) mesh in the cod end, and a $1.3 \mathrm{~cm}$ ( $0.5 \mathrm{inch})$ liner. Some crabs within coastal bays were collected in a $30.5 \mathrm{~m}(100 \mathrm{ft}) \times$ $1.8 \mathrm{~m}(6 \mathrm{ft})$ seine net with a $0.6 \mathrm{~cm}(0.25 \mathrm{inch})$ ace mesh central bag. Additionally, 5 sites in Virginia were sampled via trawl in 1994 and 1995: portions of Chincoteague Bay, a site near Kiptopeke National Wildlife Refuge, a site near Wachapreague, a site near Occahannock, and off Cape Charles within Chesapeake Bay.

In Virginia (VA), crabs were collected by several methods. In October 1993, 1994, 1996, and throughout 1997, crabs were taken with commercial crab pots from 2 reference locations adjacent to the Delmarva Peninsula: Red Bank and Hungars Creeks. In October 1993, additional samples were obtained from crab pots set in Chincoteague Bay, Mattawoman Creek, Nassawadox Creek, Wachapreague Creek, and Wachapreague Inlet. Crab pot samples were biased towards mature crabs. Broad-scale sampling within lower Chesapeake Bay was done in conjunction with the Virginia Institute of Marine Science (VIMS) Trawl Survey (April through December 1996 and 1997), and the VIMS Blue Crab Dredge Survey (part of the Chesapeake Bay Stock Assessment Program, November through March 1996 and 1997). Trawls consisted of

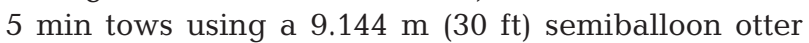
trawl (Marinivich Gulf shrimp trawl) with a $38.1 \mathrm{~mm}$ (1.5 inch) stretch mesh body, a $9.05 \mathrm{~mm}$ (0.75 inch) cod end, and a $6.35 \mathrm{~mm}$ (0.25 inch) mesh cod-end liner with attached tickler chain (0.375 inch link). Dredge tows consisted of a $1.83 \mathrm{~m}$ wide Virginia crab dredge fitted with $1.25 \mathrm{~cm}$ (0.5 inch) Vexar mesh dragged on the bottom for 1 min at 3 knots. For the 1997 collection, up to 60 crabs from each trawl or dredge sample were examined for dinoflagellates. All crabs over 28 to $30 \mathrm{~mm}$ carapace width (CW) were sampled from the high salinity sites in the VIMS Dredge Survey. After 1994, low salinity locations (e.g., York River near West Point) were not sampled for the disease, but several crabs ( $\mathrm{n}=60$ to 75 ) were later sampled from the mouths of the York and James Rivers. Temperature and salinity were recorded for each station in the Trawl and Dredge Surveys. Due to variations in sampling gear and effort, pot and trap collections from the lower Chesapeake Bay were biased toward mature crabs, whereas trawl collections from coastal bays of Maryland were biased toward immature crabs.

A series of crab samples was collected during spring and summer months from 1994 to 1997 in Delaware 
(DE) in conjunction with the DE Division of Fish and Wildlife Blue Crab Survey. Sampled sites included Indian River, Rehoboth Bay, and Delaware Bay. Sam-

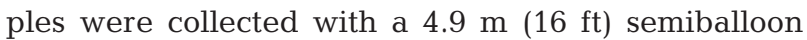
otter trawl using a $3.6 \mathrm{~cm}$ (1.4 inch) stretch mesh body with a $1.3 \mathrm{~cm}$ (0.5 inch) knotless stretch mesh liner in the cod end.

Samples of blue crabs were also obtained from various coastal bays or tributaries of New Jersey, North Carolina, South Carolina, Georgia, Mississippi, Louisiana, Texas, and the Atlantic Ocean off MD. These crabs were collected with a dredge, trawl, traps, or pots.

Water salinity and temperature were recorded, when provided, for sites where crabs were collected in coastal bays of MD. For data analyses, salinity was divided into 5 groups: $11-15,16-20,21-25,26-30$, and $31-35 \%$; temperature was also divided into 5 groups: $3-9,10-16,17-23,24-30$, and $31-37^{\circ} \mathrm{C}$. CW was measured as the longest distance between epibranchial spines. Crabs were divided into 6 size groups: $3-30$, 31-60, 61-90, 91-120, 121-150, and >150 mm CW for data analyses. Sex and maturity were recorded; male crabs under $90 \mathrm{~mm} \mathrm{CW}$ were considered immature (Millikin \& Williams 1984). The molt stage (postmolt, intermolt, premolt) of some crabs was noted from visual examination of the propodus of the 5th leg (swimmer), and the flexibility of the carapace.

Hemolymph was assayed using the methods described by Messick (1995). In some cases, crabs were shipped live to Oxford, MD; in other cases, hemolymph samples were removed and fixed on site and sent to Oxford for processing. Crabs were bled from arthrodial membranes using a $1 \mathrm{cc}$ insulin syringe with a $1.0 \mathrm{~cm}, 27$ or $28 \mathrm{ga}$ needle. Hemolymph was allowed to adhere to acidcleaned, $0.1 \% \mathrm{w} / \mathrm{v}$ poly-L-lysine-coated microscope slides for $20 \mathrm{~s}$ to $5 \mathrm{~min}$ longer times reflect observing cells live, under an inverted microscope with Hoffman modulation or phase contrast optics. Hemolymph samples were fixed in Bouin's fluid and stained with Mayer's hematoxylin and eosin (H\&E) (Luna 1968). Mean intensity and prevalence are defined in Margolis et al. (1982). Briefly, intensity is the number of individuals of a particular parasite species in each infected host. We estimated intensity by counting at least 300 cells per hemolymph preparation, divided the number of individual parasites by the total number of cells (parasites + hemocytes) counted, and multiplied by 100 . The mean intensity of infection for a sample was calculated using infected hosts only. Prevalence was determined as the number of infected crabs divided by the total number of crabs sampled and expressed as a percentage. For some analyses, infections were further divided into light (0.3 to 3.0 parasites per 100 host cells), moderate (3.1 to 10 parasites per 100 host cells), or high (>10 parasites per 100 host cells) intensity categories. Intensity is not density, which is the number of individuals of a parasite species per unit volume; cell densities were obtained for selected samples using a hemacytometer (Shields \& Squyars 2000).

Mortality rates in infected crabs were assayed in naturally infected and inoculated crabs held at $20^{\circ} \mathrm{C}$ in $24 \%$ seawater. Naturally infected crabs $(\mathrm{n}=22)$ were held individually for observation. Previously assayed naive crabs $(\mathrm{n}=15)$ had $100 \mu \mathrm{l}$ of infected hemolymph containing $10^{2}$ plasmodia $\mathrm{ml}^{-1}$ injected directly into the axillae of the 5th leg. Both male and female crabs were inoculated ( $\mathrm{n}=12,3$, respectively). The onset and course of infections were monitored via weekly hemolymph assays as described above. Uninfected control crabs were not included, as the initial purpose of this ancillary study was to examine infection dynamics; controlled mortality experiments have been reported elsewhere (Messick et al. 1999, Shields \& Squyars 2000).

Additional crustacean species were assayed for Hematodinium spp. infections. These included the following: 25 mud crabs Hexapanopeus angustifrons, Neopanope sayi, and Panopeus herbstii from the coastal bays of MD, and Core Sound, North Carolina; 13 green crabs Carcinus maenus from the coastal bays of MD and Long Island Sound, New York; 9 rock crabs Cancer irroratus from the coastal bays of $\mathrm{MD}$, and the Atlantic Ocean off MD; 29 lady crabs Ovalipes ocellatus from the coastal bays of MD; over 140 Callinectes similis from coastal bays of MD, VA, North Carolina, and Georgia; 7 grass shrimp Paleomonetes spp. from Core Sound, North Carolina; and 77 unidentified gammaridean amphipods from the coastal bays of MD. In November 1994 and January 1995, 13 C. irroratus and 5 Portunus gibbesii from lower Chesapeake Bay were examined. In June 1996, 13 C. irroratus, 9 O. ocellatus, and 1 Libinia emarginata from Wachapreague Inlet, VA, were examined. Crab species were collected and assayed for Hematodinium spp. in hemolymph, as described above. Due to their small size, amphipods and grass shrimp were fixed in Bouin's fluid and processed for histological examination by routine methods (Johnson 1980, Howard \& Smith 1983).

Data from crabs collected from the coastal bays of MD were analyzed using factorial analysis of variance (ANOVA) techniques (SAS 1999). The analyses tested the effects of host characteristics (size, sex, molt stage) and physical characteristics (water salinity and temperature) on the prevalence and mean intensity of Hematodinium sp. infections; percent intensity was square root transformed (as appropriate for percentages). The large number of stations and repeated sampling allowed reasonable sample sizes for estimates of mean prevalence for ANOVA. Observations with missing data were omitted from the analyses. Effects were considered significant at $\mathrm{p} \leq 0.05$. 


\section{RESULTS}

\section{Distribution}

Hemolymph samples from over 13000 blue crabs were examined for Hematodinium sp. infections. Collections were comprised of 4830 crabs from coastal bays of MD; 1542 from coastal bays of VA; 5076 from lower Chesapeake Bay; 695 from coastal bays of DE; and 1138 from other coastal areas from New Jersey to Texas.

Hematodinium sp. infections were widespread along the mid-Atlantic seaboard. The parasite was found in blue crabs from New Jersey to Florida, and along the Gulf coast in Texas (Fig. 1, Table 1). The widespread sampling effort indicated the parasite was present in many locations. A consistent pattern in prevalence was not observed among sampling locations and sampling times. Due to difficulty obtaining multiple samples, some areas were not sampled repeatedly; thus, areas such as Louisiana and Mississippi may be underrepresented.

Hematodinium sp. was not found in crabs from the upper and northeastern reaches of Chesapeake Bay where salinities were below $18 \%$, but was more prevalent in the higher salinity waters in the lower portions of Chesapeake Bay (Table 2). Prevalences varied in these locations over sampling periods. Seasonal variation in prevalence was observed in lower portions of Chesapeake Bay, but Hematodinium sp. was rarely found in crabs from western portions of the lower bay (Fig. 2).

Coastal bays on the Delmarva Peninsula had high prevalences of Hematodinium sp. infections, especially during autumn months. In coastal bays of DE, prevalences ranged from 0 to $69 \%$ with a mean of $36.3 \%$ ( $\pm 23.6 \mathrm{SD}, \mathrm{n}=11$ samples) during autumn. Many samples had high intensity infections (Table 3). Prevalences for crabs collected from coastal bays of VA varied with location and season, with higher prevalences during autumn. From September through December 1992 to 1998, prevalence ranged from 0 to $95 \%$ with a mean of $32( \pm 30 \mathrm{SD}, \mathrm{n}=22$ samples) (Table 4).

Table 1. Location, prevalence, and mean intensity of Hematodinium sp. infections in blue crabs sampled along the Atlantic and Gulf coasts of USA with water salinity, temperature, sample number, and collection date. Prev. = prevalence, $\mathrm{nr}=$ not recorded

\begin{tabular}{|c|c|c|c|c|c|c|}
\hline Site & $\begin{array}{c}\text { Date } \\
(\mathrm{mo} / \mathrm{d} / \mathrm{yr})\end{array}$ & $\mathrm{n}$ & $\begin{array}{l}\text { Prev. } \\
(\%)\end{array}$ & $\begin{array}{l}\text { Intensity } \\
(\%)\end{array}$ & $\begin{array}{l}\text { Salinity } \\
(\%)\end{array}$ & $\begin{array}{l}\text { Temp. } \\
\left({ }^{\circ} \mathrm{C}\right)\end{array}$ \\
\hline Stone Harbor, NJ & 09/15/98 & 34 & 9 & 23 & 32 & 28 \\
\hline Atlantic Ocean, MD & 09/12/96 & 9 & 22 & 72 & 34 & $\mathrm{nr}$ \\
\hline Atlantic Ocean, MD & $10 / 22 / 96$ & 16 & 0 & & 33 & $\mathrm{nr}$ \\
\hline Core Sound, NC & $06 / 20 / 95$ & 27 & 0 & & 30 & 25 \\
\hline Jones Bay, NC & $09 / 12 / 95$ & 63 & 0 & & 14 & 24 \\
\hline Roanoke Sound, NC & 07/16/96 & 49 & 0 & & 28 & 25 \\
\hline Newport River, NC & 07/18/96 & 47 & 0 & & 25 & 31 \\
\hline Core Sound, NC & 07/19/96 & 76 & 5 & 28 & 33 & 27 \\
\hline Ashley River, SC & 07/23/96 & 20 & 5 & 64 & 22 & 29 \\
\hline Wando River, SC & 07/23/96 & 20 & 10 & 49 & 22 & 29 \\
\hline Charleston Harbor, SC & 07/23/96 & 20 & 30 & 58 & 27 & 28 \\
\hline North Edisto System, SC & 07/31/96 & 25 & 0 & & 22 & 31 \\
\hline Lower Ashley River, SC & 08/05/97 & 27 & 15 & 2 & 18 & 28 \\
\hline Port Royal Sound, SC & 03/18/98 & 22 & 0 & & 25 & 13 \\
\hline Wando River, SC & 07/07/98 & 14 & 0 & & 24 & 30 \\
\hline Ashley \& Wando Rivers, SC & 09/10/98 & 16 & 12 & 80 & 25 & 28 \\
\hline St. Simons Island, GA & $11 / 09 / 96$ & 23 & 4 & 2 & 27 & 19 \\
\hline St. Simons, GA & 05/24/99 & 85 & 6 & 57 & 28 & 26 \\
\hline $\begin{array}{l}\text { Wassaw, Ossabaw, St. Simons, } \\
\text { Cumberland, St. Andrew, GA }\end{array}$ & 06/02/99 & 105 & 22 & 24 & $24-34$ & 27 \\
\hline $\begin{array}{l}\text { Wassaw, Ossabaw, Sapelo, } \\
\text { St. Simons, St. Andrew, GA }\end{array}$ & 07/13/99 & 217 & 5 & 25 & $24-34$ & 29 \\
\hline $\begin{array}{l}\text { Ossabaw, Sapelo, } \\
\text { St. Simons, GA }\end{array}$ & 08/10/99 & 36 & 0 & & $22-34$ & 31 \\
\hline Ft. Pierce, FL & 07/08/99 & 45 & 40 & 13 & 27 & 35 \\
\hline Davis Bayou, MS & $09 / 15 / 97$ & 32 & 0 & & 18 & 27 \\
\hline Grand Isle, LA & 09/15/97 & 32 & 0 & & 23 & 32 \\
\hline Caillou Lake, LA & $11 / 09 / 96$ & 30 & 0 & & 25 & 23 \\
\hline Corpus Christi Bay, TX & $11 / 20 / 96$ & 23 & 9 & 48 & 30 & 23 \\
\hline Corpus Christi Bay, TX & $07 / 23 / 97$ & 8 & 0 & & 33 & 30 \\
\hline Aransas Bay, TX & $10 / 30 / 96$ & 17 & 6 & 15 & 30 & $\mathrm{nr}$ \\
\hline
\end{tabular}


Table 2. Location, prevalence, and mean intensity of Hematodinium sp. infections in blue crabs sampled within Chesapeake Bay with water salinity, temperature, sample number, and collection date. Samples for 1996 VA sites are shown in Fig. 2. Prev. = prevalence, $\mathrm{nr}=$ not recorded

\begin{tabular}{|c|c|c|c|c|c|c|}
\hline Site & $\begin{array}{c}\text { Date } \\
(\mathrm{mo} / \mathrm{d} / \mathrm{yr})\end{array}$ & $\mathrm{n}$ & $\begin{array}{c}\text { Prev. } \\
(\%)\end{array}$ & $\begin{array}{c}\text { Intensity } \\
(\%)\end{array}$ & $\begin{array}{c}\text { Salinity } \\
(\% \text { ) }\end{array}$ & $\begin{array}{l}\text { Temp. } \\
\left({ }^{\circ} \mathrm{C}\right)\end{array}$ \\
\hline Nanticoke River, MD & 03/22/95 & 116 & 0 & & 14 & 12 \\
\hline Tangier Sound, MD & $11 / 04 / 93$ & 68 & 0 & & 12 & 16 \\
\hline Pocomoke Sound, MD & 10/27/93 & 75 & 0 & & 10 & 11 \\
\hline Smiths Beach, VA & $09 / 29 / 94$ & 19 & 0 & & & \\
\hline Occahannock, VA & $07 / 15 / 94$ & 26 & 0 & & 15 & 30 \\
\hline Occahannock, VA & $08 / 15 / 94$ & 20 & 0 & & & \\
\hline Occahannock, VA & $09 / 15 / 94$ & 34 & 0 & & 17 & 25 \\
\hline Occahannock, VA & $10 / 18 / 94$ & 13 & 0 & & 16 & 17 \\
\hline Occahannock, VA & $11 / 14 / 94$ & 19 & 0 & & 18 & 14 \\
\hline Occahannock, VA & $07 / 13 / 95$ & 57 & 0 & & 19 & 29 \\
\hline Occahannock, VA & $08 / 23 / 95$ & 60 & 0 & & 21 & 29 \\
\hline Occahannock, VA & 09/19/95 & 31 & 0 & & 21 & 24 \\
\hline Occahannock, VA & $10 / 23 / 95$ & 55 & 0 & & 21 & 19 \\
\hline Nassawadox Creek, VA & $08 / 23 / 93$ & 24 & 0 & & 20 & \\
\hline Nassawadox Creek, VA & $08 / 23 / 93$ & 21 & 14 & $\mathrm{nr}$ & & \\
\hline Nassawadox Creek, VA & $08 / 25 / 93$ & 30 & 3 & $\mathrm{nr}$ & 20 & \\
\hline Hungars Creek, VA & $08 / 24 / 93$ & 21 & 5 & $\mathrm{nr}$ & 20 & \\
\hline Hungars Creek, VA & $09 / 28 / 94$ & 44 & 2 & 9 & & \\
\hline Hungars Creek, VA & $09 / 30 / 94$ & 19 & 0 & & & \\
\hline Hungars Creek, VA & $10 / 27 / 94$ & 24 & 8 & 32 & & \\
\hline Hungars Creek, VA & $11 / 14 / 94$ & 24 & 0 & & & \\
\hline Hungars Creek, VA & $12 / 07 / 94$ & 18 & 0 & & & \\
\hline Hungars Creek, VA & $04 / 25 / 96$ & 22 & 0 & & & \\
\hline Hungars Creek, VA & 05/17/96 & 40 & 0 & & & \\
\hline Hungars Creek, VA & $06 / 11 / 96$ & 40 & 0 & & & \\
\hline Hungars Creek, VA & $07 / 29 / 96$ & 57 & 0 & & & \\
\hline Hungars Creek, VA & $08 / 21 / 96$ & 43 & 0 & & & \\
\hline Hungars Creek, VA & $09 / 25 / 96$ & 37 & 0 & & & \\
\hline Hungars Creek, VA & $10 / 24 / 96$ & 43 & 2 & $\mathrm{nr}$ & & \\
\hline Hungars Creek, VA & $11 / 20 / 96$ & 21 & 0 & & & \\
\hline Hungars Creek, VA & $05 / 02 / 97$ & 40 & 0 & & & \\
\hline Hungars Creek, VA & $09 / 25 / 97$ & 40 & 18 & 42 & & \\
\hline Hungars Creek, VA & $10 / 30 / 97$ & 47 & 0 & & & \\
\hline Hungars Creek, VA & $05 / 12 / 98$ & 20 & 5 & $\mathrm{nr}$ & & \\
\hline Hungars Creek, VA & $06 / 17 / 98$ & 22 & 0 & & & \\
\hline Hungars Creek, VA & $07 / 20 / 98$ & 27 & 0 & & & \\
\hline Hungars Creek, VA & 09/17/98 & 32 & 0 & & & \\
\hline Hungars Creek, VA & $10 / 06 / 98$ & 28 & 7 & $\mathrm{nr}$ & & \\
\hline Mattawoman Creek, VA & $08 / 24 / 93$ & 21 & 0 & & & \\
\hline York River, VA & $01 / 03 / 95$ & 24 & 0 & & & \\
\hline York River, VA & $12 / 14 / 94$ & 94 & 0 & & & \\
\hline York River, VA & $12 / 19 / 95$ & 60 & 0 & & & \\
\hline Cape Charles, VA & $07 / 15 / 94$ & 44 & 0 & & 20 & 30 \\
\hline Cape Charles, VA & $08 / 15 / 94$ & 34 & 0 & & & \\
\hline Cape Charles, VA & $09 / 15 / 94$ & 30 & 3 & 22 & 21 & 23 \\
\hline Cape Charles, VA & $10 / 18 / 94$ & 47 & 4 & 13 & 22 & 16 \\
\hline Cape Charles, VA & $11 / 14 / 94$ & 14 & 0 & & 23 & 15 \\
\hline Cape Charles, VA & $07 / 13 / 95$ & 62 & 11 & 43 & 23 & 29 \\
\hline Cape Charles, VA & $08 / 23 / 95$ & 31 & 10 & 37 & 23 & 27 \\
\hline Cape Charles, VA & $09 / 19 / 95$ & 21 & 29 & 29 & 24 & 22 \\
\hline Cape Charles, VA & $10 / 23 / 95$ & 60 & 18 & 38 & 25 & 18 \\
\hline Lower bay, VA & $11 / 29 / 94$ & 62 & 0 & & & \\
\hline Lower bay, VA & $12 / 21 / 94$ & 34 & 0 & & & \\
\hline Lower bay, VA & $03 / 06 / 95$ & 19 & 0 & & & \\
\hline Lower bay, VA & $12 / 18 / 95$ & 98 & 2 & 14 & & \\
\hline Tue Point, York River, VA & $10 / 06 / 94$ & 35 & 0 & & & \\
\hline James River, VA & $12 / 30 / 99$ & 67 & 0 & & & \\
\hline
\end{tabular}

Salinity

Within the coastal bays of MD, the distribution of Hematodinium sp. was significantly associated with high salinities ( $\mathrm{p}<$ 0.0001) (Fig. 3). Prevalence was highest in the 26 to $30 \%$ salinity range with $38 \%$ infected $(\mathrm{n}=2130)$. No crabs collected from salinities $<11 \%$ o $(\mathrm{n}=45)$ were found with infections. Mean intensity did not vary significantly among infected crabs collected from the various salinity groups ( $p=0.36)$ in coastal bays of MD. In Chesapeake Bay and its western tributaries, prevalence was zero in salinities below $18 \%$ ( $\mathrm{n}=833$ ) (Table 2). No infected crabs were collected at salinities below 18\% from other areas sampled along the Atlantic and Gulf coasts (Table 1).

\section{Hydrography}

In the coastal bays of MD, prevalence of Hematodinium sp. varied significantly among 20 trawl stations ( $\mathrm{p}<0.0001)$. Stns T01 to T07, located north of Ocean City Inlet, were among the stations with the lowest prevalence. Additionally, Stns T12 and T17 in the upper reaches of Newport Bay and Green Run Bay had relatively lower prevalences of infections than nearby stations (Fig. 4). Average salinities at Stns T05 and T12 were comparatively lower than nearby stations (Fig. 4).

\section{Seasonality}

Monthly or periodic sampling from 1992 to 1998 in coastal bays of MD showed that prevalence and intensity of Hematodinium sp. infections followed a seasonal trend, with a distinct peak in late autumn ( $p<0.001$, Fig. 5). Although infection prevalences gradually increased throughout the summer, they peaked in late autumn, with a sharp increase in average prevalence from 49 to $81 \%$ from October to November (Figs. 5 \& 6). Prevalences precipitously declined in January, and were virtually undetectable from March through May. In June, infections became apparent, and mean prevalence rose to $20 \%$ (Figs. 5 \& 6). Prevalences within 
Winter 1996

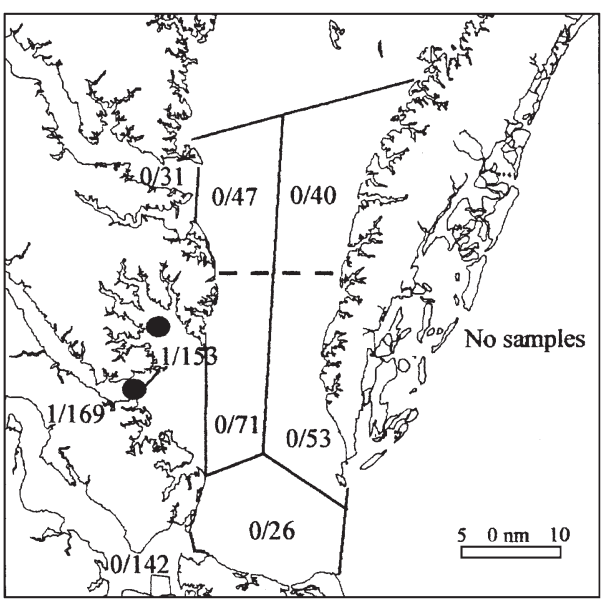

Summer 1996

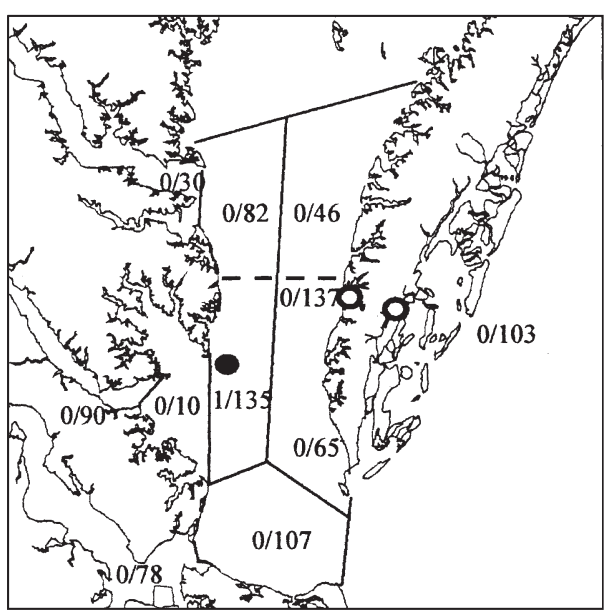

Fig. 2. Distribution and prevalence of Hematodinium sp. infections in blue crabs sampled in lower Chesapeake Bay during winter, spring, summer, and autumn 1996 and autumn 1997. Sample size was 2870 crabs exclusive of those given for Hungars and Red Bank Creeks in Tables 2 \& 4 . Numbers indicate infected crabs vs total number sampled. Aggregate locations correspond to geographic strata used by VIMS Trawl Survey. (O) Locations where infected crabs found; (O) reference locations: Hungars Creek and Red Bank Creek

Chesapeake Bay also varied by season, with peaks during autumn (Fig. 2). Mean intensities within MD coastal bays followed a seasonal trend, but peaked earlier in September (Fig. 6). The prevalence of Hematodinium sp. varied significantly among crabs collected
Spring 1996

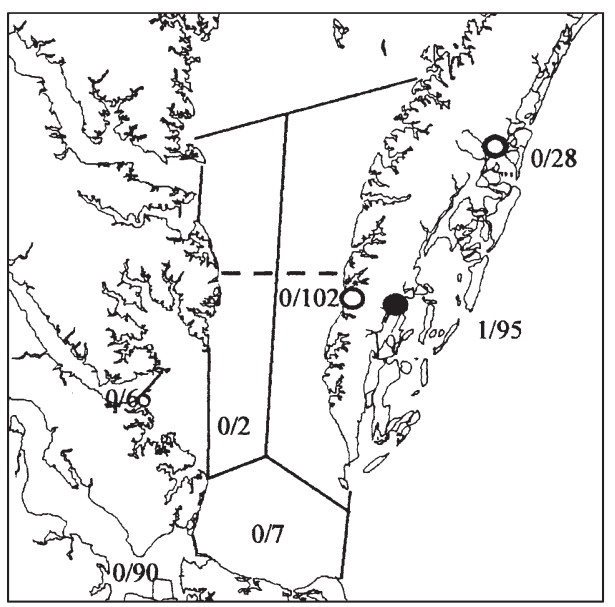

Autumn 1996

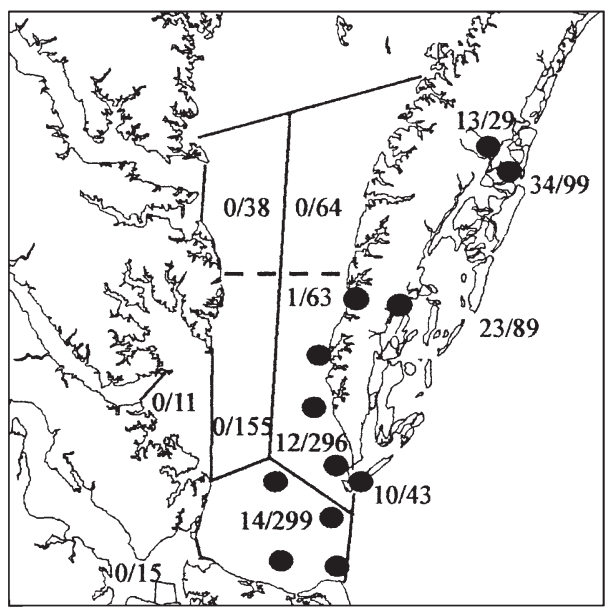

Autumn 1997

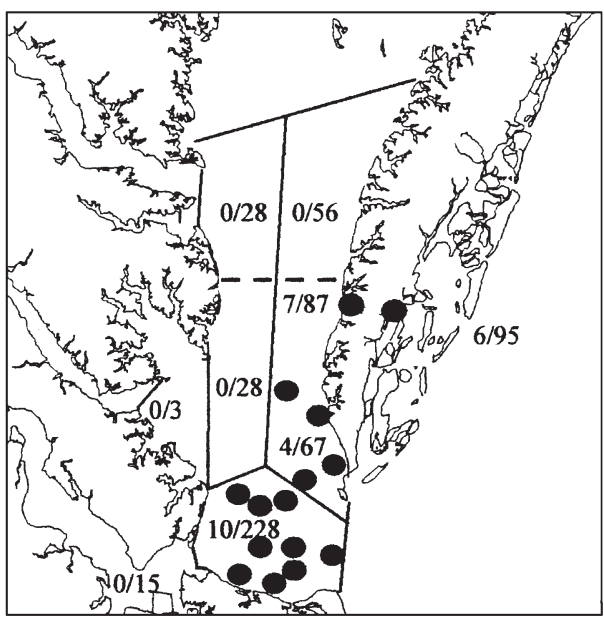

at different temperatures $(p<0.0001)$ (Fig. 7). The prevalence of infections was significantly higher in crabs from 3 to $9^{\circ} \mathrm{C}$ waters $(\mathrm{p}<0.0001)$ than any other temperature range. Mean intensity varied significantly among crabs collected at different temperature ranges 
Table 3. Prevalence and mean intensity of Hematodinium sp. infections in blue crabs sampled from Delaware (DE) embayments (Indian River, Rehoboth Bay, and Delaware Bay) with sample number and collection date. Prev = prevalence, Int = mean intensity

\begin{tabular}{|c|c|c|c|c|c|c|c|c|c|}
\hline \multirow{2}{*}{$\begin{array}{l}\text { Date } \\
(\mathrm{mo} / \mathrm{d} / \mathrm{yr})\end{array}$} & \multicolumn{3}{|c|}{ Indian River } & \multicolumn{3}{|c|}{ Rehoboth Bay } & \multicolumn{3}{|c|}{ Delaware Bay } \\
\hline & Prev. (\%) & $\mathrm{n}$ & Int. (\%) & Prev. (\%) & $\mathrm{n}$ & Int. (\%) & Prev. (\%) & $\mathrm{n}$ & Int. (\%) \\
\hline 07/14/94 & 8 & 77 & 16 & & & & & & \\
\hline 09/01/94 & 32 & 37 & 23 & 22 & 58 & 32 & & & \\
\hline 10/01/94 & & & & 13 & 37 & 30 & 65 & 62 & 33 \\
\hline 05/01/95 & & & & 0 & 5 & 0 & & & \\
\hline 06/01/95 & 24 & 25 & 35 & 0 & 31 & 0 & & & \\
\hline 07/01/95 & 26 & 23 & 49 & 6 & 34 & 23 & & & \\
\hline 09/01/95 & 0 & 23 & 0 & 33 & 6 & 78 & & & \\
\hline 10/01/95 & 51 & 51 & 40 & & & & & & \\
\hline 07/01/96 & 27 & 30 & 42 & 33 & 36 & 67 & & & \\
\hline 07/30/96 & & & & & & & & & \\
\hline 09/01/96 & 56 & 16 & 76 & 50 & 8 & 42 & 8 & 49 & 91 \\
\hline 07/01/97 & 28 & 29 & 75 & 31 & 29 & 48 & & & \\
\hline 09/01/97 & & & & & & & 69 & 29 & 73 \\
\hline
\end{tabular}

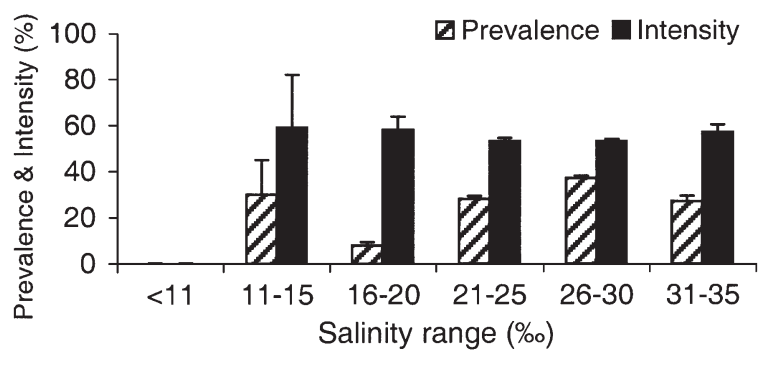

Fig. 3. Comparison of mean prevalence and mean intensity of Hematodinium sp. infections among blue crabs collected from different water salinity ranges within coastal bays of Maryland from 1992 to 1998. Error bar = SE

$(\mathrm{p}=0.004)$; but in contrast to prevalence, mean intensity was significantly lower in crabs from 3 to $9^{\circ} \mathrm{C}$ waters than crabs from 10 to $16^{\circ} \mathrm{C}(\mathrm{p}=0.02)$, 17 to $23^{\circ} \mathrm{C}(\mathrm{p}=0.02)$, and 24 to $30^{\circ} \mathrm{C}(\mathrm{p}=0.02)$ waters.

\section{Host factors}

The prevalence of Hematodinium sp. infections in coastal bays of MD varied significantly among the 6 host size classes throughout the year $(\mathrm{p}=$ $<0.0001$ ) (Fig. 8). Prevalence was significantly higher in crabs measuring $3-30 \mathrm{~mm} \mathrm{CW}$, than in those measuring 31-60, 61-90, 91-120, 121-150, or $>150 \mathrm{~mm} \mathrm{CW}(\mathrm{p}<0.005)$. Additional analysis of data collected in autumn months, when disease prevalence peaked, indicated that mean prevalences in smaller crabs measuring $\leq 60 \mathrm{~mm} \mathrm{CW}$ were significantly higher than prevalences in larger crabs measuring $>60 \mathrm{~mm} \mathrm{CW}(\mathrm{p}<0.0007)$.
Mean intensity did not vary among size classes during autumn months $(\mathrm{p}<0.20)$, nor throughout the year $(\mathrm{p}<$ $0.13)$.

In coastal bays of MD, the prevalence of Hematodinium sp. infections varied significantly between mature $(26 \%)$ and immature $(35 \%)$ crabs $(p<0.0005)$ throughout the year. Also, during autumn months when prevalence peaked, prevalence was significantly higher $(\mathrm{p}<0.0001)$ in immature crabs $(65 \%)$ than in mature crabs $(38 \%)$.
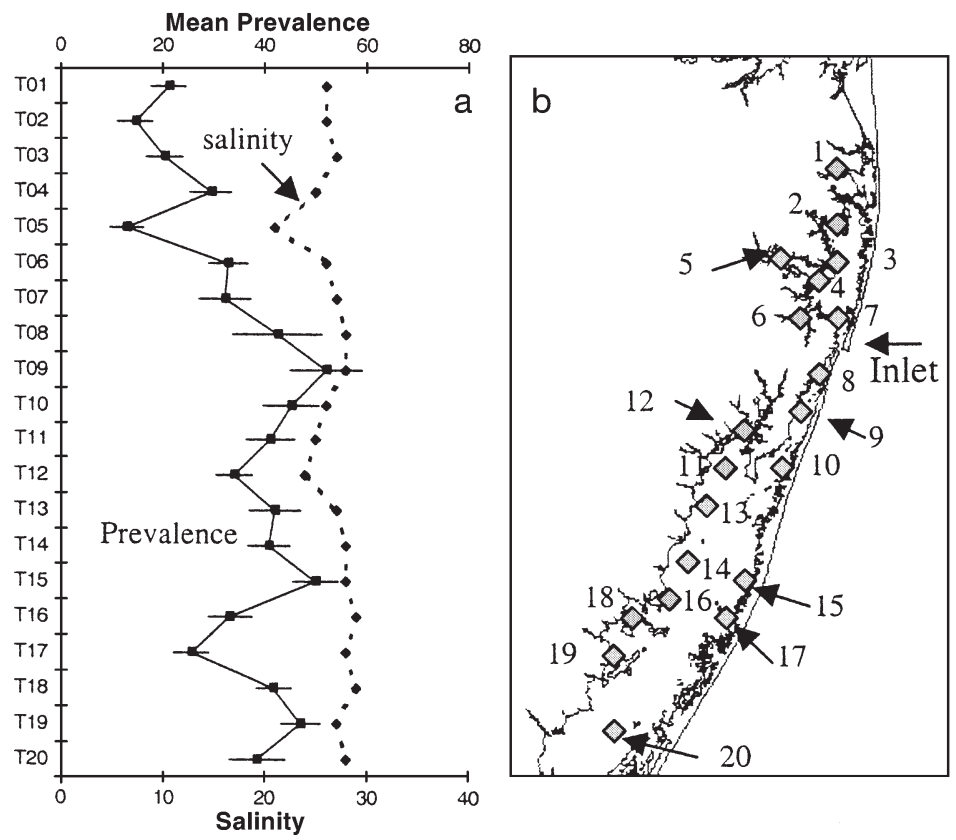

Fig. 4. (a) Mean prevalence of Hematodinium sp. infections in blue crabs and mean water salinity over time from sites within coastal bays of Maryland sampled from 1992 to 1998. Error bar = SE. (b) Location of various sites within coastal bays of Maryland sampled semi-monthly from 1992 to 1998 
Table 4. Location, prevalence, and mean intensity of Hematodinium sp. infections in blue crabs sampled from Virginia (VA) coastal bays with water salinity, temperature, sample number, and collection date. Prev. = prevalence, $\mathrm{nr}=$ not recorded

\begin{tabular}{|c|c|c|c|c|c|c|}
\hline Site & $\begin{array}{c}\text { Date } \\
\text { (mo/d/yr) }\end{array}$ & $\mathrm{n}$ & $\begin{array}{c}\text { Prev. } \\
(\%)\end{array}$ & $\begin{array}{c}\text { Intensity } \\
(\%)\end{array}$ & $\begin{array}{c}\text { Salinity } \\
(\%)\end{array}$ & $\begin{array}{c}\text { Temp. } \\
\left({ }^{\circ} \mathrm{C}\right)\end{array}$ \\
\hline Chincoteague & $10 / 08 / 92$ & 40 & 93 & 39 & & \\
\hline Chincoteague & 08/25/93 & 24 & 13 & $\mathrm{nr}$ & & \\
\hline Chincoteague & 08/25/93 & 27 & 7 & $\mathrm{nr}$ & & \\
\hline Chincoteague & $10 / 26 / 93$ & 20 & 95 & 24 & & \\
\hline Chincoteague & 06/15/95 & 81 & 44 & 35 & 23 & 23 \\
\hline Chincoteague & 07/20/95 & 34 & 59 & 43 & 29 & 29 \\
\hline Chincoteague & 08/23/95 & 28 & 43 & 29 & 33 & 26 \\
\hline Chincoteague & 09/19/95 & 27 & 67 & 48 & 29 & 20 \\
\hline Chincoteague & $10 / 23 / 95$ & 31 & 61 & 37 & 23 & 18 \\
\hline Wachapreague & $10 / 27 / 94$ & 15 & 27 & $\mathrm{nr}$ & & \\
\hline Wachapreague & 08/30/95 & 20 & 15 & 9 & 32 & 26 \\
\hline Wachapreague & $10 / 26 / 95$ & 20 & 80 & 26 & 32 & 18 \\
\hline Wachapreague & $10 / 21 / 96$ & 99 & 34 & 21 & & \\
\hline Finney Creek & $10 / 21 / 96$ & 29 & 48 & 14 & & \\
\hline Red Bank Creek & $08 / 24 / 93$ & 37 & 16 & $\mathrm{nr}$ & 30 & \\
\hline Red Bank Creek & $09 / 28 / 94$ & 47 & 6 & 36 & & \\
\hline Red Bank Creek & $09 / 30 / 94$ & 14 & 7 & 0.3 & & \\
\hline Red Bank Creek & $10 / 27 / 94$ & 108 & 7 & 40 & & \\
\hline Red Bank Creek & $11 / 14 / 94$ & 46 & 13 & 32 & & \\
\hline Red Bank Creek & $12 / 07 / 94$ & 65 & 18 & 19 & & \\
\hline Red Bank Creek & $04 / 25 / 96$ & 32 & 0 & & & \\
\hline Red Bank Creek & 05/17/96 & 36 & 3 & 5 & & \\
\hline Red Bank Creek & 06/13/96 & 27 & 0 & & & \\
\hline Red Bank Creek & 07/29/96 & 32 & 0 & & & \\
\hline Red Bank Creek & 08/21/96 & 34 & 0 & & & \\
\hline Red Bank Creek & 09/25/96 & 35 & 0 & & & \\
\hline Red Bank Creek & $10 / 24 / 96$ & 38 & 16 & 53 & & \\
\hline Red Bank Creek & $11 / 20 / 96$ & 26 & 35 & 5 & & \\
\hline Red Bank Creek & $12 / 16 / 96$ & 25 & 32 & 20 & & \\
\hline Red Bank Creek & $05 / 02 / 97$ & 45 & 13 & 23 & & \\
\hline Red Bank Creek & $09 / 25 / 97$ & 22 & 14 & 20 & & \\
\hline Red Bank Creek & $10 / 30 / 97$ & 73 & 4 & $\mathrm{nr}$ & & \\
\hline Red Bank Creek & $05 / 12 / 98$ & 40 & 7.5 & 7 & & \\
\hline Red Bank Creek & $06 / 17 / 98$ & 49 & 0 & & & \\
\hline Red Bank Creek & $07 / 20 / 98$ & 37 & 22 & 26 & & \\
\hline Red Bank Creek & 09/17/98 & 37 & 5.4 & 51 & & \\
\hline Red Bank Creek & $10 / 06 / 98$ & 52 & 13 & 39 & & \\
\hline Kiptopeke & $08 / 30 / 95$ & 19 & 58 & 23 & 31 & 25 \\
\hline Fishermans Island & $10 / 23 / 96$ & 43 & 23 & 22 & & \\
\hline
\end{tabular}

Overall, the prevalence of Hematodinium sp. infections did not vary significantly between male (34\%) and female $(33 \%)$ crabs in coastal bays of MD ( $\mathrm{p}=$ 0.40). However, during autumn 1996, in the coastal bays of VA, mature males $(25.7 \%)$ had significantly higher prevalence than mature females $(4.3 \%$ ) (chisquare, $\mathrm{p}<0.001)$. In addition, southeastern sites within Chesapeake Bay such as Cape Charles and Fishermans Island had significantly higher prevalences in males (18 to $31 \%$ ) than in females (0 to $3 \%$ ). Mean intensities did not vary significantly among mature and immature infected male and female crabs from the coastal bays of MD ( $p=0.18)$.

The molt condition of blue crabs from coastal bays of MD was not significantly associated with prevalence or intensity of Hematodinium sp. infections $(\mathrm{p}=0.52$, 0.41 , respectively). Prevalence was $15 \%$ in intermolt, $19 \%$ in postmolt, and $17 \%$ in premolt crabs. Mean intensity was $47 \%$ $(\mathrm{n}=273)$ in intermolt, 38\% $(\mathrm{n}=150)$ in postmolt, and $33 \%(\mathrm{n}=47)$ in premolt crabs. Some infected crabs molted in the laboratory $(\mathrm{n}=3)$, but changes in intensity were not examined for molting crabs.

\section{Mortality experiments}

Naturally infected crabs held in captivity suffered $100 \%$ mortality over $35 \mathrm{~d}$. The mean time to death was approximately $13 \mathrm{~d}$, and infections ranged from light to heavy intensities. Mortalities in experimentally inoculated crabs began after $17 \mathrm{~d}$, with a mean time to death of

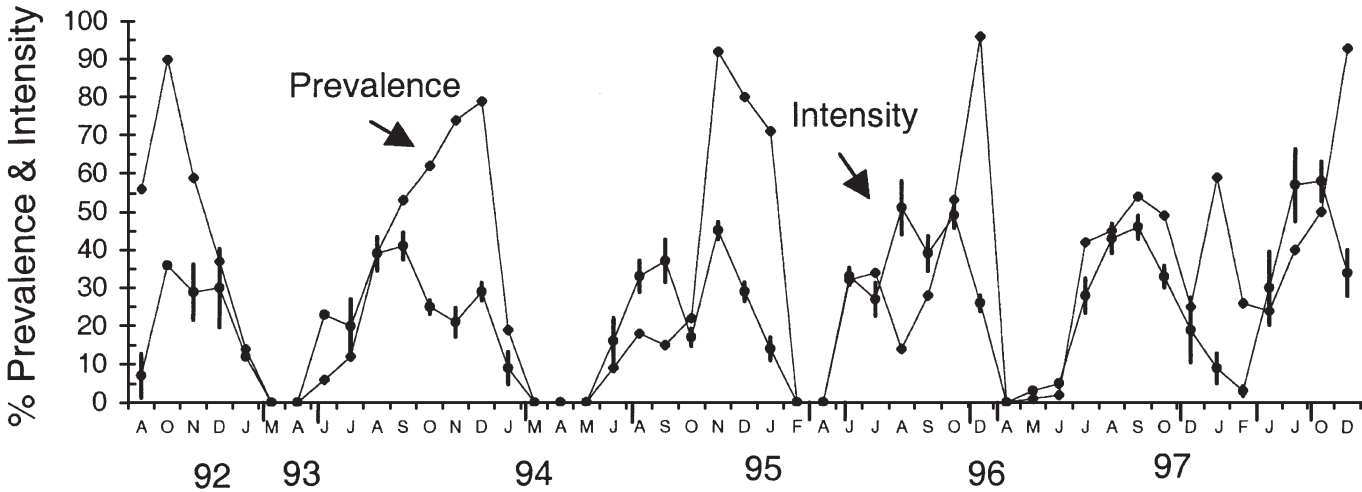

Fig. 5. Seasonal variation in mean prevalence and mean intensity of Hematodinium sp. infections in blue crabs collected semimonthly from coastal bays of Maryland from 1992 to 1997. Error bar = SE 
Fig. 6. Seasonal variation in mean prevalence and mean intensity of Hematodinium sp. infections in blue crabs from coastal bays of Maryland from 1992 to 1997 with mean monthly water temperature

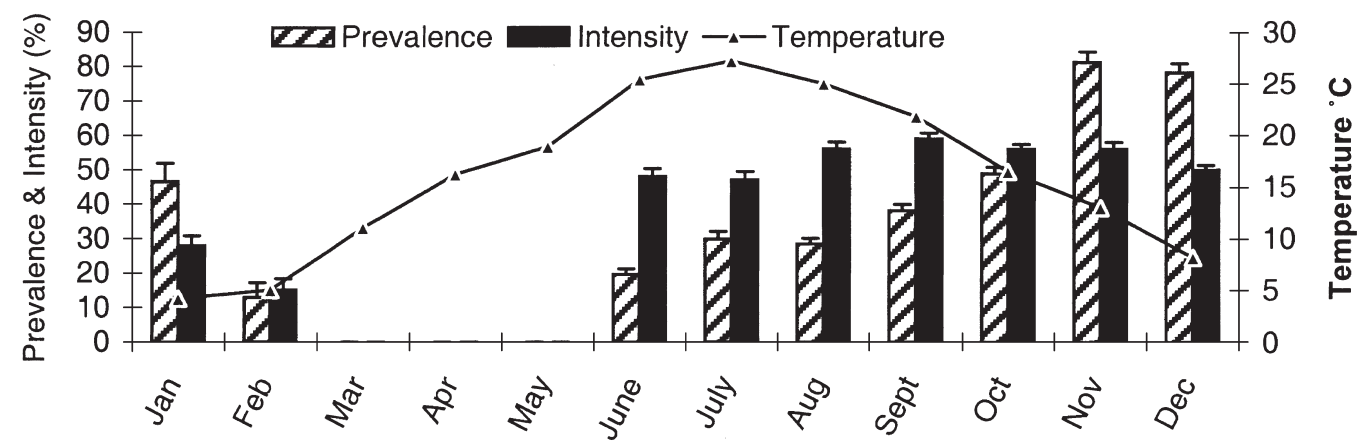

approximately $42 \mathrm{~d}$ and $100 \%$ mortality after $55 \mathrm{~d}$ (Fig. 9). Naturally infected crabs with light infections developed heavy infections over 2 to $3 \mathrm{wk}$ (Fig. 10). The proliferation of the parasite was not consistent between lightly, moderately and heavily infected crabs, and in some cases, heavily infected crabs survived as long as lightly infected crabs (Fig. 10). Crabs with high intensity infections on Day 1 likely had end-stage infections and lagged in parasite proliferation perhaps due to depleted host resources or due to chronic infections. Inocula of $10^{2}$ plasmodia successfully transmitted the parasite to naive hosts. In general, no infections were observed prior to $6 \mathrm{~d}$ post-inoculation. Light infections were observed after $13 \mathrm{~d}$ in 14 of 15 hosts. Moderate infections occurred after $16 \mathrm{~d}$, and heavy infections occurred after $30 \mathrm{~d}$.

\section{Additional parasites}

Numerous other parasites were detected in hemolymph samples of crabs collected from the coastal bays of MD. Hemocytes with cytoplasmic inclusions similar to those described as virus infections (Johnson 1985) were found in 19 crabs; a histophagous ciliate, Mesanophrys chesapeakensis (Messick \& Small 1996), was found in 4; unidentified microsporidians (Sprague 1977) were found in 28; Paramoeba perniciosa (Sprague

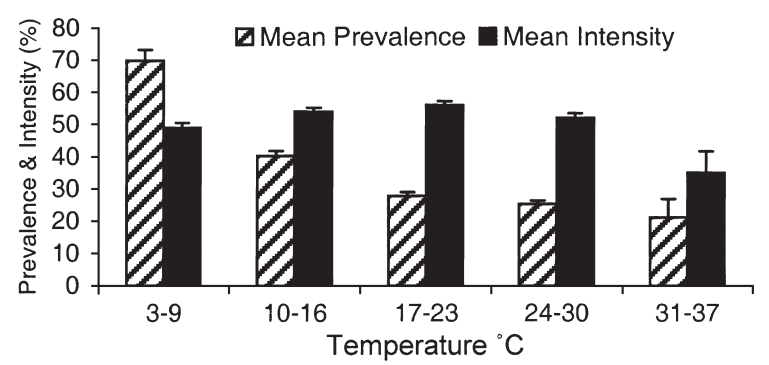

Fig. 7. Comparison of mean prevalence and mean intensity of Hematodinium sp. infections in blue crabs collected among different water temperature ranges within coastal bays of Maryland from 1992 to 1998. Error bar $=\mathrm{SE}$
\& Beckett 1966, Johnson 1977) was found in 35; and a haplosporidian-like organism (Newman et al. 1976) was found in 2 sampled crabs. Some crabs which had Hematodinium sp. infections were co-infected with other parasites, including $P$. perniciosa $(\mathrm{n}=6)$, an unidentified virus $(\mathrm{n}=1)$, unidentified microsporidians $(\mathrm{n}=3)$, and $M$. chesapeakensis $(\mathrm{n}=3)$. Although many parasites can be found in hemolymph, parasite infections were likely higher than reported since parasites may have been present in tissues other than hemolymph.

\section{Alternate hosts}

Hematodinium sp. infections were detected in $8 \%$ of mud crabs, $8 \%$ of Carcinus maenus collected from the coastal bays of MD, and $0.7 \%$ C. similis collected from Georgia. A sample of gammarid amphipods collected on 22 April 1994 from MD coastal bays was not infected, but $8.5 \%$ of a sample collected on 21 August 1996 were infected with a Hematodinium-like dinoflagellate. Infections were not observed in Paleomonetes spp., Cancer irroratus, Ovalipes ocellatus, Portunus gibbesii, nor Libinia emarginata. In some cases, collections were not made in autumn when parasite prevalence peaks in blue crabs.

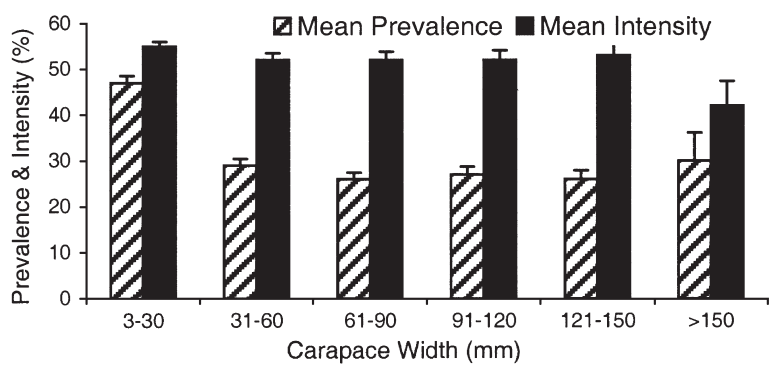

Fig. 8. Comparison of mean prevalence and mean intensity of Hematodinium sp. infections among blue crabs of different size ranges collected within coastal bays of Maryland from 1992 to 1998. Error bar = SE 


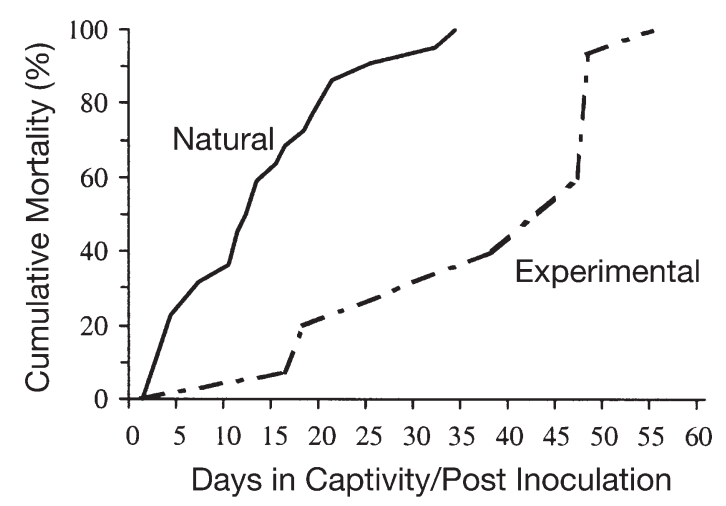

Fig. 9. Mortality over time in blue crabs held at $20^{\circ} \mathrm{C}$ in $24 \%$ seawater for up to $60 \mathrm{~d}$; naturally infected crabs $(\mathrm{n}=22)$ and crabs experimentally inoculated with Hematodinium sp. plasmodia $(n=15)$

\section{DISCUSSION}

Hematodinium sp. infections in blue crabs are widespread along the Atlantic and Gulf coasts of the United States. Retrospective analysis of landings data shows a marked decline in the fishery which corresponds to initial reports of heavy mortalities and high prevalences of infected crabs in coastal bays of MD and VA (Messick 1994) (Fig. 11). Hematodinium sp. infections are highly pathogenic in blue crabs; in the present study, naturally infected crabs suffered $100 \%$ mortality over $35 \mathrm{~d}$ at 20 to $24^{\circ} \mathrm{C}$. In a separate study, mortality rates were greater than $86 \%$ in experimentally infected crabs compared to $20 \%$ in uninfected controls (Shields \& Squyars 2000). Natural infections appear to become latent at lower temperatures, but when crabs are held at higher temperatures, infections become active (Messick et al. 1999). The wide distribution, high prevalence, and high mortality rate of infected crabs indicate that Hematodinium sp. is a significant potential source of mortality in juvenile and adult blue crabs and may severely impact coastal fisheries during seasonal epizootics.

MD, VA, North Carolina, Louisiana, and other coastal states sustain major blue crab fisheries. Some areas of the USA have reported a downward trend in crab fisheries (Evans 1998, Guillory et al. 1998, Steele \& Bert 1998). It is difficult to estimate mortality due to disease, and to separate disease effects from the numerous other sources of natural mortality; therefore, mortality is typically assigned a constant value in models designed to estimate crab stocks. Considering the widespread distribution and virulence of Hematodinium sp. in the blue crab, the development of differential models for exploitation should consider regional (coastal vs riverine) and environmental factors (reports of epizootics, salinity increases due to drought, warm
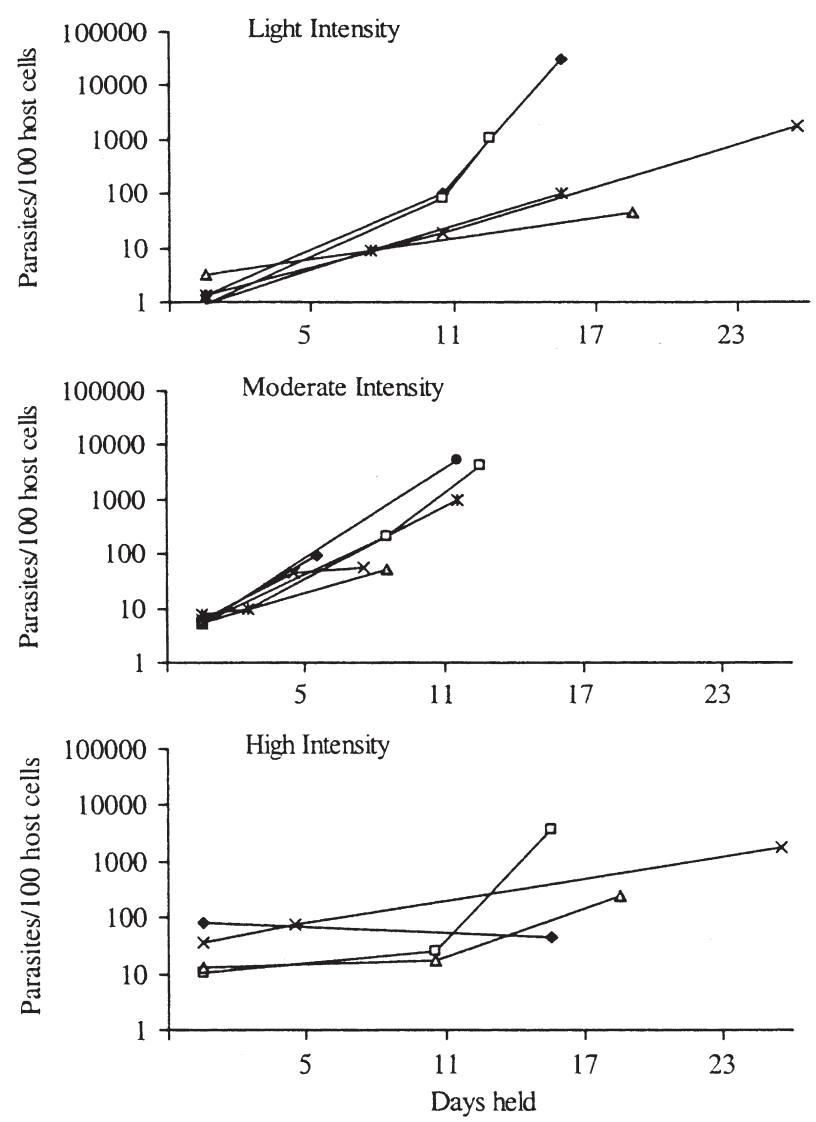

Fig. 10. Proliferation of Hematodinium sp. in naturally infected blue crabs with light (0.3 to 3.0 parasites/100 host cells), moderate (3.1 to 10 parasites/100 host cells) or heavy (>10 parasites/100 host cells) infections. Each line represents an individual crab. Note log scale for parasite proliferation

winters that may enhance parasite survival) that may affect blue crab stocks.

Hematodinium sp. occurs in crabs from waters above $11 \%$ salinity (Newman \& Johnson 1975), including areas within Chesapeake and Delaware Bays; therefore, the disease is not strictly a high-salinity coastal bay phenomenon. Most of the crabs collected from the lower Chesapeake Bay were in, or adjacent to, sanctuaries designed to protect pre-ovigerous and spawning crabs. The presence of infections in adult female crabs (1 to $13 \%$ ) from Chesapeake Bay, coupled with greater prevalences in juveniles and crabs from higher salinities (Messick 1994), may allow the disease to threaten reproduction in sanctuaries during major epizootics and to impact survival of juvenile crabs protected as recruits to the fishery. It is unknown why juvenile crabs are more prone to Hematodinium sp. infections than larger crabs. High population densities during the annual transient profusion in the juvenile population may predispose them to the parasite, or mature crabs may appear less susceptible due to their removal by the fishery or by premature removal by infections. 


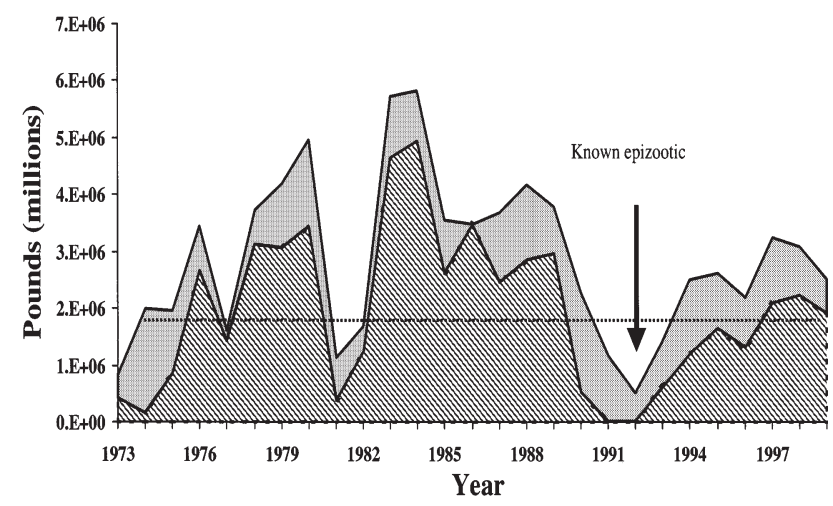

Fig. 11. Annual landings of blue crabs from seaside coastal bays of Maryland () and Virginia (imiv) shown with average yearly landings for Virginia (..........). Landings data from Virginia Marine Resources Commission, Stock Assessment Program

Hydrographic features may contribute to epizootics of Hematodinium sp. in blue crabs. In the coastal bays of MD, the prevalence of Hematodinium sp. varied by location in relation to salinity, and to general drainage or flushing patterns. Stations with some of the lowest prevalences were located north of the ocean inlet, and in tributaries (Fig. 4). The greatest drainage of this system is into the northern portions (Sieling 1960), i.e., increased flushing via this drainage pattern may partially explain the lower prevalence of infections in the northern coastal bays of MD. Hydrographic conditions may contribute to high prevalences of infections in crabs from Chincoteague Bay, Wachapreague, and Red Bank Creek. Limited flow of water through these shallow, high salinity lagoons may focus or amplify the infectious stages of the parasites. Hydrographic conditions are important features in epizootics of egg predatory nemerteans, rhizocephalan castrators, and other dinoflagellate infections of crabs and lobsters (Sloan 1984, Meyers et al. 1987, 1990, Kuris et al. 1991, Field et al. 1992). In Alaskan waters, hydrographic conditions such as fjords with shallow sills coupled with seasonal increases in water temperature contribute to epizootics of several parasites (Sloan 1984, Meyers et al. 1990, Kuris et al. 1991). Coastal bays along the Delmarva Peninsula appear ideal for the proliferation of epizootics of Hematodinium sp. and Paramoeba perniciosa (Shields 1994). The region includes relatively closed crab populations, based on low immigration and emigration rates of juveniles and adults (Kuris \& Lafferty 1992), relatively high salinity with little water exchange between the open ocean and backwaters, and stressful conditions such as high temperatures and seasonal hypoxia. Similar conditions exist in many small estuaries along the midAtlantic and southeastern USA.
Hematodinium sp. infections showed marked autumnal seasonality in blue crabs. Distinct seasonal peaks in Hematodinium spp. infections are apparent in other crustacean fisheries. The seasonal prevalence of Hematodinium sp. in Alaskan Tanner crabs Chionoecetes bairdi peaks in summer, declines in winter, and increases again in the spring (Eaton et al. 1991, Love et al. 1993). Hematodinium sp. in Necora puber from France causes mortalities in winter, with prevalence peaks in May and June (Wilhelm \& Boulo 1988, Wilhelm \& Miahle 1996). Hematodinium sp. in Cancer pagurus is found throughout the year (Latrouite et al. 1988). Infections in the Norway lobster Nephrops norvegicus peak from April to May (Field et al. 1998) with latent infections from July to December (Appleton \& Vickerman 1998). Infected Jonah crabs C. borealis were found throughout the year (MacLean \& Ruddell 1978), but sample frequency was too low to determine seasonal patterns. The prevalence of a dinoflagellatelike protozoan reported in Alaskan shrimps Pandalus platyceros and $P$. borealis peaks in winter and the onset of infections are associated with colder temperatures (Meyers et al. 1994).

Several days to months are required for dinoflagellate infections in some crustaceans to progress from early subpatent infections to detectable infections (Meyers et al. 1987, Field \& Appleton 1996, Appleton \& Vickerman 1998, Field et al. 1998). The ability to detect infections may be delayed in naturally infected crabs due to low initial parasite numbers. Sensitivity (percentage of infected crabs exhibiting detectable parasites in the hemolymph) was relatively low (30 to $35 \%$ ) in crabs inoculated with $10^{3}$ or $10^{5}$ parasites after $2 \mathrm{wk}$ but increased to 80 to $85 \%$ after 26 to 32 d (Shields \& Squyars 2000). In this study (Figs. 5 \& 6), no patent infections were found during the early spring; low temperatures during winter may cause the parasite to become subpatent and there may be a delay in patency despite the warmer spring water temperatures. Although water temperatures are highest during summer, parasite prevalences were rather steady. Parasite proliferation may be hindered by high water temperatures during summer. Data collected from estuaries in Georgia during June, July, and August 1999 indicate the prevalence of infections decreased at higher summer temperatures (Table 1). The prevalence of infections in coastal bays of MD was highest during autumn when water temperatures were relatively cooler; this season may provide the optimal temperatures for parasite proliferation. Prevalence declined precipitously during winter months when water temperatures dropped. A water temperature of $9^{\circ} \mathrm{C}$ reduced parasite intensity in naturally infected crabs held for $73 \mathrm{~d}$ (Messick et al. 1999). Water temperature and parasite proliferation rates at these temperatures likely induce the 
seasonality of Hematodinium sp. infections in blue crabs. There was no indication that infection seasonality was due to seasonal fluctuations in salinity.

Crab size is a determining factor in the prevalence and distribution of Hematodinium sp. in blue crabs. Smaller crabs are more prone to infection than mature crabs and were more prevalent than larger crabs in autumn samples; crab size may also influence the seasonality of infections. Juvenile crabs molt more frequently than larger crabs. Host defense mechanisms may be stressed by ecdysis, rendering juvenile crabs more susceptible to invading organisms. Newly molted Tanner crabs are more likely to harbor detectable dinoflagellate infections than pre-molt crabs (Meyers et al. 1990, Eaton et al. 1991), and infections in the Norway lobster Nephrops norvegicus peak from March to May, the period of highest molting activity (Field et al. 1992). Molting may predispose crabs to invasion by certain parasites by allowing entry through breaks in the cuticle (Couch \& Martin 1982, Meyers et al. 1990, Morado \& Small 1994, Hoeg 1995). Although no significant variation in prevalence was found in crabs at different molt stages in this study, sample numbers were relatively low. Additionally, although prevalence of disease was $0 \%$ in coastal bays of MD during May, when molting peaked, prevalence increased to $30 \%$ just 1 mo later in June.

Infections of Hematodinium-like dinoflagellates in other crustaceans suggest the presence of alternate or reservoir hosts. Hudson \& Shields (1994) speculated that amphipods may serve as intermediate or reservoir hosts for infections. Since blue crabs eat amphipods and other crabs, predation or cannibalism may perhaps spread infections. We have not, however, been able to transmit Hematodinium spp. infections via feeding (Hudson \& Shields 1994) or co-habitation (Messick et al. 1999). Parasites found in mud crabs, green crabs, Callinectes similis, or amphipods may be the same species as that found in the blue crab. Morphologically, the parasites show similarities in size, form, and nuclear staining characteristics. At present, molecular studies (Hudson \& Adlard 1996) and ultrastructural investigations have not examined the type species from the locality of the type host. Clearly, additional assessments of green crabs for Hematodinium sp. would facilitate our understanding of the taxonomy of this important group of pathogens.

In summary, Hematodinium sp. infections in blue crabs from the USA are widely distributed, seasonal, and influenced by location, salinity, and host size. Numerous questions remain about how environmental and host factors modulate parasite prevalence, proliferation, and virulence. These variables are likely influenced by physiological characteristics of the host, the parasite, or they may synergistically influence infections.
Acknowledgements. The following people helped collect crabs: Stu Michels-DE Division of Fish and Wildlife; Jim Casey, Steve Doctor, Al Wesche, Craig Weedon, Rudy Lukacovic, Larry Pieper-MD Department of Natural Resources; Phil Wirth-University of MD Eastern Shore; Dorothy Howard-NOS, Oxford; Elaine Haueber, Chris Squyars, Pat Geer, Marcel Montane, Kyrie Bernstein, Landon Ward, Mike Seebo, Todd Matheson, and others of the VIMS Trawl and Dredge Surveys; Vince Guillory_Louisiana Department of Wildlife and Fisheries; Tom Wagner-Texas Parks and Wildlife; Dwayne Roberson-Georgia Department of Natural Resources; Richard Lee, Jason Harrison, and Ayana McCoySkidaway Oceanographic Institute, GA; Dean AhrenholzNMFS Beaufort Laboratory; Elaine Andersen-New Jersey fisherwoman; Larry DeLancey-SC Marine Fisheries Division; Lynn Henry, David Taylor, Sean McKenna, Mike Pulley, Lele Judy, Wayne Cuthrell, Parks Lewis, and Joe AndrewsNC Division of Marine Fisheries; Mike Brainard-Mississippi Department of Marine Resources; Robin Overstreet-Gulf Coast Research Laboratory-University of Southern Mississippi; and many others. We also thank the following for processing samples: Carol McCollough, Sue Tyler, Charlie Gieseker, Jeff Clemmer, and Dorothy Howard of the Cooperative Oxford Laboratory; Nita Walker and Rita Crockett of VIMS; and Richard Lee, Jason Harrison, and Ayana McCoy of the Skidaway Oceanographic Institute, GA. This work was supported, in part, by NOAA, Saltonstall-Kennedy Grants NA66FD0018 and NA76FD0148, VA Sea Grant R/MG94-3 to JDS, and MD Sea Grant No. RL1AACM and partial support by MD Department of Natural Resources to GAM. This is Contribution No. 2329 from the Virginia Institute of Marine Science.

\section{LITERATURE CITED}

Abbe GR, Stagg C (1996) Trends in blue crab (Callinectes sapidus Rathbun) catches near Calvert Cliffs, Maryland, from 1968 to 1995 and their relationship to the Maryland commercial fishery. J Shellfish Res 15:751-758

Appleton PL, Vickerman K (1998) In vitro cultivation and development cycle in culture of a parasitic dinoflagellate (Hematodinium sp.) associated with mortality of the Norway lobster (Nephrops norvegicus) in British waters. Parasitology 116:115-130

Chatton E, Poisson R (1931) Sur l'existance, dans le sang des crabs, de peridiniens parasites: Hematodinium perezi n.g., n.sp. (Syndinidae). CR Seances Soc Biol Fil 105:553-557

Couch JA, Martin S (1982) Protozoan symbionts and related diseases of the blue crab, Callinectes sapidus Rathbun from the Atlantic and Gulf coasts of the United States. In: Perry HM, Van Engel WA (eds) Proceedings of the blue crab colloquium, Biloxi, MS. Gulf States Marine Fisheries Commission, Ocean Springs, MS, p 71-81

Eaton WD, Love DC, Botelho C, Meyers TR, Imamura K, Koeneman T (1991) Preliminary results on the seasonality and life cycle of the parasitic dinoflagellate causing bitter crab disease in Alaskan Tanner crabs (Chionoecetes bairdi). J Invertebr Pathol 57:426-434

Evans C (1998) Conservation and management of the blue crab fishery in Georgia. J Shellfish Res 17:451-458

Field RH, Appleton PL (1996) An indirect fluorescent antibody technique for the diagnosis of Hematodinium sp. infection of the Norway lobster Nephrops norvegicus. Dis Aquat Org 24:199-204

Field RH, Chapman CJ, Taylor AC, Neil DM, Vickerman K (1992) Infection of the Norway lobster Nephrops norvegicus by a Hematodinium-like species of dinoflagellate on 
the west coast of Scotland. Dis Aquat Org 13:1-15

Field RH, Hills JM, Atkinson JA, Magill S, Shanks AM (1998) Distribution and seasonal prevalence of Hematodinium sp. infection of the Norway lobster (Nephrops norvegicus) around the west coast of Scotland. ICES J Mar Sci 55: 846-858

Guillory V, Perry H, Steele P, Wagner T, Hammerschmidt P, Heath S, Moss C (1998) The Gulf of Mexico blue crab fishery: historical trends, status, management, and recommendations J Shellfish Res 17:395-403

Hoeg JT (1995) The biology and life cycle of the Rhizocephala (Cirripedia) J Mar Biol Assoc UK 75:517-550

Howard DW, Smith CS (1983) Histologic techniques for marine bivalve mollusks. US Dept Commerce, NOAA Tech Memo NMFS-F/NEC-25

Hudson DA, Adlard RD (1996) Nucleotide sequence determination of the partial SSU rDNA gene and ITS1 region of Hematodinium cf. perezi and Hematodinium-like dinoflagellates. Dis Aquat Org 24:55-60

Hudson DA, Shields JD (1994) Hematodinium australis n. sp., a parasitic dinoflagellate of the sand crab Portunus pelagicus from Moreton Bay, Australia. Dis Aquat Org 19: 109-119

Hudson DA, Hudson N, Shields JD (1993) Infection of Trapezia spp. (Decapoda: Xanthidae) by Hematodinium sp. (Duboscquodinida: Syndinidae): a new family record of infection. J Fish Dis 16:273-276

Johnson JA Jr, Green DP, Martin RE (1998) Industry perspectives: the hard blue crab fishery-Atlantic and Gulf. J Shellfish Res 17:371-374

Johnson PT (1977) Paramoebiasis in the blue crab Callinectes sapidus. J Invertebr Pathol 29:308-320

Johnson PT (1980) Histology of the blue crab, Callinectes sapidus: a model for the Decapoda. Praeger, New York

Johnson PT (1985) Blue crab (Callinectes sapidus Rathbun) viruses and the diseases they cause. In: Perry HM, Malone RF (eds) National symposium on the soft-shelled blue crab fishery. Gulf Coast Research Laboratory, Biloxi, MS, p 13-19

Johnson PT (1986) Parasites of benthic amphipods: dinoflagellates (Duboscquodinida: Syndinidae). Fish Bull 84: 605-614

Kuris AM, Lafferty KD (1992) Modeling crustacean fisheries: effects of parasites on management strategies. Can J Fish Aquat Sci 49:327-336

Kuris AM, Blau SF, Paul AJ, Shields JD, Wickham DE (1991) Infestation by brood symbionts and their impact on egg mortality in the red king crab, Paralithodes camtschatica, in Alaska: geographic and temporal variation. Can J Fish Aquat Sci 48:559-568

Latrouite D, Morizur Y, Noel P, Chagot D, Wilhelm G (1988) Mortalite du tourteau Cancer pagurus provoqueé par le dinoflagelle parasite: Hematodinium sp. Int Counc Explor Sea CM-ICES/K:32

Lipcius RN, Van Engel WA (1990) Blue crab population dynamics in Chesapeake Bay: variation in abundance (York River, 1972-1988) and stock-recruit functions. Bull Mar Sci 46:180-194

Love DC, Rice SD, Moles DA, Eaton WD (1993) Seasonal prevalence and intensity of bitter crab dinoflagellate infection and host mortality in Alaskan Tanner crabs Chionoecetes bairdi from Auke Bay, Alaska, USA. Dis Aquat Org 15:1-7

Luna LG (1968) Manual of histologic staining methods of the Armed Forces Institute of Pathology, 3rd edn. McGrawHill Book Co, New York

MacLean SA, Ruddell CL (1978) Three new crustacean hosts for the parasitic dinoflagellate Hematodinium perezi (Dinoflagellata: Syndinidae). J Parasitol 64:158-160
Margolis L, Esch GW, Holmes JC, Kuris AM, Schad GA (1982) The use of ecological terms in parasitology (report of an ad hoc committee of the American Society of Parasitologists). J Parasitol 68:131-133

Messick GA (1994) Hematodinium perezi infections in adult and juvenile blue crabs Callinectes sapidus from coastal bays of Maryland and Virginia, USA. Dis Aquat Org 19: 77-82

Messick GA (1995) Laboratory techniques to detect parasites and diseases of blue crabs, Callinectes sapidus. In: Stolen JS, Fletcher TC, Smith A, Zelikoff JT, Kaattari SL, Anderson RS, Söderhäll K, Weeks-Perkins BA (eds) Techniques in fish immunology - 4. SOS Publications, Fair Haven, NJ, p 187-198

Messick GA, Small EB (1996) Mesanophrys chesapeakensis n. sp., a histophagous ciliate in the blue crab, Callinectes sapidus, and associated histopathology. Invertebr Biol 115: $1-12$

Messick GA, Jordan SJ, van Heukelem WF (1999) Salinity and temperature effects on Hematodinium sp. in the blue crab Callinectes sapidus. J Shellfish Res 18:657-662

Meyers TR, Koeneman TK, Botelho C, Short S (1987) Bitter crab disease: a fatal dinoflagellate infection and marketing problem for Alaskan Tanner crabs Chionoecetes bairdi. Dis Aquat Org 3:195-216

Meyers TR, Botelho C, Koeneman TM, Short S, Imamura K (1990) Distribution of bitter crab dinoflagellate syndrome in southeast Alaskan Tanner crabs, Chionoecetes bairdi. Dis Aquat Org 9:37-43

Meyers TR, Lightner DV, Redman RM (1994) A dinoflagellate-like parasite in Alaskan spot shrimp Pandalus platyceros and pink shrimp P. borealis. Dis Aquat Org 18:71-76

Meyers TR, Morado JF, Sparks AK, Bishop GH, Pearson T, Urban D, Jackson D (1996) Distribution of bitter crab syndrome in Tanner crabs (Chionoecetes bairdi, C. opilio) from the Gulf of Alaska and the Bering Sea. Dis Aquat Org 26:221-227

Millikin MR, Williams AB (1984) Synopsis of biological data on the blue crab, Callinectes sapidus Rathbun. US Dept Commerce, NOAA Tech Rep NMFS 1, FAO Fish Synop No 138

Morado JF, Small EB (1994) Morphology and stomatogenesis of Mesanophrys pugettensis n. sp. (Scuticociliatida: Orchitophryidae), a facultative parasitic ciliate of the Dungeness crab, Cancer magister (Crustacea: Decapoda). Trans Am Microsc Soc 113:343-364

Newman MW, Johnson CA (1975) A disease of blue crabs (Callinectes sapidus) caused by a parasitic dinoflagellate, Hematodinium sp. J Parasitol 63:554-557

Newman MW, Johnson CA, Pauley GP (1976) A Minchinialike haplosporidan parasitizing blue crabs, Callinectes sapidus. J Invertebr Pathol 27:311-315

Rugolo LJ, Knotts KS, Lange AM, Crecco VA (1998) Stock assessment of Chesapeake Bay blue crab (Callinectes sapidus Rathbun). J Shellfish Res 17:906-930

SAS (1999) Using Statview. SAS Institute Inc, Cary, NC

Shields JD (1992) Parasites and symbionts of the crab Portunus pelagicus from Moreton Bay, eastern Australia. J Crustac Biol 12:92-100

Shields JD (1994) The parasitic dinoflagellates of marine crustaceans. Annu Rev Fish Dis 4:241-271

Shields JD, Squyars CM (2000) Mortality and hematology of blue crabs, Callinectes sapidus, experimentally infected with the parasitic dinoflagellate Hematodinium perezi. Fish Bull 98:139-152

Sieling FW (1960) The resources of Worcester County coastal waters. Ref. no 60-10, MD Department of Research and 
Education, Chincoteague Bay Station, Snow Hill, MD

Sloan NA (1984) Incidence and effects of parasitism by the rhizocephalan barnacle, Briarosaccus callosus Boschma, in the golden king crab, Lithodes aequispina Benedict, from deep fjords in northern British Columbia, Canada. J Exp Mar Biol Ecol 84:111-131

Sprague V (1977) Classification and phylogeny of the Microsporidia. In: Bulla LA, Cheng TC (eds) Comparative pathobiology: treatises, Vol 2. Systematics of the Microsporidia. Plenum Press, New York, p 1-30

Sprague V, Beckett RL (1966) A disease of blue crabs (Callinectes sapidus) in Maryland and Virginia. J Invertebr Pathol 8:287-289

Editorial responsibility: Timothy Flegel,

Bangkok, Thailand
Steele P, Bert TM (1998) The Florida blue crab fishery: history, status, and management. J Shellfish Res 17:441-449

Taylor DM, Khan RA (1995) Observations on the occurrence of Hematodinium sp. (Dinoflagellata: Syndinidae), the causative agent of bitter crab disease in Newfoundland snow crab (Chionoecetes opilio). J Invertebr Pathol 65: $283-288$

Wilhelm G, Boulo V (1988) Infection de l'etrille Liocarcinus puber (L.) par un dinoflagelle parasite de type Hematodinium sp. Int Counc Explor Sea CM-ICES/K:41

Wilhelm G, Mialhe E (1996) Dinoflagellate infection associated with the decline of Necora puber crab populations in France. Dis Aquat Org 26:213-219

Submitted: July 19, 1999; Accepted: August 29, 2000 Proofs received from author(s): November 6, 2000 